germinal entity never changes its nature. In its earliest period of existence it is absolutely invisible. It only becomes visible when empowered by hatching to receive and assimilate food from the glaire of the egg; and this function proves the pre-existence of a vital elementary organization. How, then, does this vital entity receive its first food? in other words, how does the primordium grow, and become visible? This question may, I think, be solved by a reference to the vegetable kingdom. For example, if we examine the flower of a common pea, we find that it grows from a peduncle or umbilical cord, and that the flower consists of a calyx of four leaves, a corolla of three or four leaves, nine stamina, and a pistillum which grows from the parent peduncle, and forms the centre of the flower. If we dissect the pistillum we find, that from its root upwards it is hollow, and contains the rudiments of the future peas, and that each of these rudimentary peas grows from a filament or umbilical cord extending from the inner surface of its containing cavity,-the future pod, - to the eye of the pea, and thence to the point of the rostellum of the corcle, - the root of the future plant. Again: if we remove the husk or outer membrane from a seasoned pea, and afterwards place the denuded pea in water, we notice that the rostellum - or root, begins to grow before the plumula - or stem of the future plant; and every person acquainted with the anatomy of the pea knows that the pointed extremity of the rostellum is connected with the hilium or eye, where the first food of the plant and last of the pea are conveyed to them. Again: if we place a number of denuded peas in water, in separate glasses, some having the point, some the half, and some the whole of the rostellum removed, we find that all of the peas having no rostellum have no power to grow, and die in a few days, whilst the others retain a portion of that power for a few days only, and then die. These experiments show that the rostellum of the pea is organized, and that its pointed extremity is empowered to imbibe matte through the hilium whenever the pea begins to grow. Now if the germ of the chick be analogous to that of a pea, all the mystery which veils the hatching process is removed. From the earliest period of existence the primordium must have an umbilical cord constructed to absorb matter through the mouth of its amniotic sac, " whenever it begins to emerge into the active state; and when this takes place, the extremity of the umbilical cord, like the extremity of the rostellum of the pea, must pass through the mouth of the sac, and descend towards the occulus ovi, over which it subsequently grows, to form the blastoderma.

The blastoderma is the structure which first appears visible in the hatching process, and from being an expansion of the umbilical cord of the primordium, it proves the pre-existence, and likewise illustrates the functions, of that cord. When the cord emerges from the orifice of the amniotic sac, it grows, and forms a membrane shaped like a double night-cap, with one part placed within the other. The inner fold covers the outer surface of the amniotic sac, and the outer fold covers the substances of the egg and the inner surface of the chorion. The outer fold grows faster, and becomes more extensive than the inner fold, and is divided by anatomists into a nutritious and a respiratory portion. These folds give to the primordium the appearance of being formed out of their wrinkles.t I have already stated, that about the twentyfourth hour of hatching the respiratory portion of the blastoderma becomes studded with red-coloured granules, which gradually disappear with the cooling of the egg; that about the twenty-seventh hour of hatching an undulating or vibratory motion exists in the primordium; and that about an hour afterwards the punctum saliens or heart is in action, and the blood seen flowing through the vessels of the respiratory membrane, and changing its colour from a dark crimson to a bright scarlet hue. These phenomena show that the blastoderma is an organic structure, and that it performs very important functions in the animal economy, for it absorbs the food, and prepares the blood for the growth of the primordium, and hence constitutes the alpha and omega of its assimilating system.

Here I bring my remarks on the hatching of the egg of the common fowl to a close for the present. As the hatching season has again arrived, and as many professional and scientific readers of THE LANCET may keep fowls, I hope that some of those gentlemen will try the following experiments, (for I have no opportunity of trying them myself, and afterwards publish in its pages the result of their labours:- Cover

* This sac is not the Graafian vesicle of authors, for that vesicle is analo gous to the yelk.

+ See THE LANCET, April 4th, 1846, p. 381, for the opinions of others upon these wrinkles. ixteen hens' nests with the following materials-namely, four nests with sheep's wool, four with cotton wool, four with wilk, and four with tow; then place in each nest ten eggs, of one breed and age, and slightly cover them with a porucking hens, all of one breed, upon the several nests, and otice the results. Again: try the same experiments with

Upper Fitzroy-street, Fitzroy-square, May, 1848.

\section{CLINICAL REMARKS ON THE DISEASES OF WOMEN.}

By JAMES MILMAN COLEY, M.D.,

EMBER OF THE ROYAL COLLEGE OF PHYSICIANS IN LONDON, ETC., PHP SIClAN TO THE WESTEKN DISFENSARY, AND SENIOR PHYSICLAN TO THE OYAL PIMLICO DISPENSARY.

On Lymphatic Tumour in the Female Breast.

TuE absorbent vessels on the upper part of the breast leading to the axilla are subject to a disease characterized by a painful, ender, and irritable swelling, and consisting of several cordlike indurations, at some times disposed in parallel rows, at ther times connected after the manner of an anastomosis. ther parts of the breast are occasionally the seats of this ffection; and in whatever situation it occurs the swelling is transverse, following the direction of the absorbents towards the axilla. On a superficial examination the tumour may escape detection; but it can always be discovered by taking the suspected part between the fingers and thumb. When the pain and tenderness are extreme, the absorbent glands in the axilla, and more rarely below the clavicle, become enarged from irritation. These glandular enlargements always disappear after the original disease has subsided: the lymphatic swelling in the breast also frequently retires, leaving no vestige behind it. In extreme cases, however, a permanent thickening takes place, occasioned by the deposit of lymph in the cellular membrane. This disease usually attacks females between the ages of fifteen and thirty-five, and is liable to recur repeatedly, where the constitution is in the peculiar state predisposing to it. The condition to which $I$ allude is that of comparative emaciation, accompanied with irregular or deficient menstruation, depression of spirits, and general debility. Hence suckling and chlorotic women are most frequently the subjects of attack. In some instances the patients are inclined to attribute the origin of the disease to external violence: in the majority of instances, however, if not in all, it has appeared to me to proceed from imperfect menstruation. In one instance I had an opportunity of examining the uterus in a patient labouring under this disease, when I found the posterior portion adjoining the cervix in a state of congestion, presenting to the finger a doughy or anasarcous feeling. The size of the tumour in the mamma varies from that of an almond to that of an adult thumb; and the pain and tenderness attending it are of a remittent character. In some rare cases it attains nearly the size of a pullet's egg in large and plethoric mammæ.

One of these tumours, which was removed at the earnest solicitation of the patient, who had suffered severely from repeated attacks of the disease, was found, on examination, to consist of thickening of the coats of the lymphatic vessels, imbedded in a stratum of condensed cellular membrane.

As this affection is dependent on the state of the general health, and particularly on an imperfect performance of the periodical function of the uterus, its duration is uncertain. I have known it return in the same individual, and as repeatedly subside under proper treatment; and it must be observed that the swelling, pain, and tenderness, in most cases, uniformly undergo an increase on the approach of menstruation. The natural termination of the disease in severe cases, when the special treatment adapted for its cure is not employed, is in a painful and obstinate ulceration, which in external appearance has a considerable resemblance to that proceeding from scrofula, the absorbent glands in the vicinity being enlarged, tender, and painful, and the discharge copious. Before ulceration commences, the cellular membrane subjacent to the skin becomes indurated; this induration is gradually softened, the skin assumes an inflamed appearance, and a small, chronic, scrofula-like abscess is the result. The ulcer which follows resists all local treatment until the proper constitutional remedy is adopted.

Diagnosis.-The discrimination of this disease from others resembling it is not difficult. From the chronic, mammary 
tumour, described by Sir A.P. Cooper, ${ }^{*}$ it may be distinguished by the pain and extreme tenderness, by the vitiated state of the patient's health, by the absence of lobes and of any cyst, and by the disease invading the breasts of suckling women more frequently than those of virgins. The condition of the uterus, too, is widely different: in the mammary tumour, a state of excitement prevails; in the lymphatic tumour, a deficient circulation takes place in that organ, manifested by the discharge of an imperfect secretion, or false membrane, from its mucous surface.

From the irritable tumour, and neuralgic state of the breast, this disease may be known by the transverse, parallel, or anastomosing, cord-like bands, which are always present, by the remission of the pain and tenderness, and by the latter symptoms being confined, as far as regards the breast, to the immediate locality of the tumour. The diagnosis in the examination of very large breasts is sometimes difficult. (See Case 6.)

Treatment.-When the pain and tenderness are excessive, leeches and evaporating poultices may be applied to the integuments over the tumour. In general it will be found unnecessary to adopt any local remedies, as the pain is not acute, but usually of an aching kind, like that accompanying rheumatism or phlegmasia dolens. The patient should take some preparation of iron twice daily, have the bowels relieved by an aloetic aperient, if needful, and use a generous diet, and gentle exercise in the open air. Should suckling have been long continued, the infant should be weaned, especially if the patient has been the mother of many children. By attending to these directions the tumour will entirely disappear in a few weeks, or all uneasiness will be so far removed that the patient will feel no inconvenience from it, unless the constitutional and uterine derangement should recur.

Case 1.-1815, Oct. 14: Miss W-, aged twenty-four, had during several years experienced at intervals severe pain and tenderness in a tumour situated at the upper part of the breast. The swelling was about an inch and a half in length, and half an inch in width, and was found to consist of clusters of parallel absorbents, greatly thickened and adherent to the adjacent cellular membrane, which had become indurated by lymph, deposited in consequence of the repeated attacks of vaseular excitement.

Four leeches were by my direction applied to the painful part, and compound iron mixture, with infusion of quassia, was prescribed.

On Nov. 7 th, the pain and tenderness were entirely removed, though the permanent thickening of the cellular membrane continued.

CASE 2.-1829, June 2: Miss $\mathrm{H}-$, aged eighteen, complained of a painful and irritable swelling in the upper portion of the breast, towards the axilla, which she supposed had proceeded from a blow received four years previously. The tumour, when taken between the fingers and thumb, felt like a mass of hardened absorbents, disposed in rows like strings. The pain was of the rheumatic kind, extended down the arm, and was much increased by any manual examination.

Teeches and an evaporating lotion were prescribed, and pills composed of two grains of sulphate of irort and one grain of aloes were administered twice a day.

On the 29 th, I found the tumour completely removed, together with the pain and soreness; and the general health was restored.

Case 3.-1830, Oct. 30: A poor woman, mother of five children, one of whom, five mouths old, she was then suckling, complained several months of a lymphatic swelling in the upper part of one of her breasts. The swelling resembled to the touch a small bundle of cords, and had become more painful and sore than usual during the last two months. She had not menstruated since her confinement.

I directed her to take two grains of sulphate of iron, and one grain and a half of aloes, twice a day, and to wean her child. In a few weeks the disease in the breast subsided.

1832, Jan. 9: This patient again applied for my advice, stating that she was suckling another child six months old, and that the painful tumour in the breast had begun to return. The chalybeate medicine was repeated, and in the course of a few weeks all symptoms of disease disappeared, notwithstanding that she continued to suckle her child during the progress of the case.

CASE 4.-1831, Feb. 26: J.S-, aged 28, mother of two children, the younger of whom was seven years old, com-

* Illustrations of the Diseases of the Breast. By Sir A. P. Cooper, Bart. plained for several months of an aching pain and great tender. ness in the upper part of the left breast, where I discovered a tumour, consisting of a congeries of thickened lymphatic vessels. Menses regular, but very deficient. Bowels consti; pated. I prescribed the sulphate of iron with aloes twice a-day, and on the 19th of March $I$ found the lymphatic character of the tumour more distinct; the adjoining fulness, arising from chronic inflammation in the cellular substance, having disappeared.

April 9th.-The breast quite restored and the menstrual discharge improved.

Oct. 22 nd.-The disease returned to an increased extent; the breast being almost covered with indurated lymphatics resembling cords, passing towards the axilla. The disease had also migrated from one breast to the other, affecting them alternately, and ultimately returning to the one originally attacked. The medicine being repeated, the disease was again removed at the end of five weeks, leaving no vestige of the swelling.

1833, Feb. 2: Extreme pain and tenderness without swell. ing again attacked the left breast, and the menses had become deficient. The medicine was repeated, and the patient was again rendered free from all symptoms at the end of a few weeks; no mark of the local disease remaining.

1835, Nov. 3: the painful lymphatic tumour returned a fourth time, together with the tenderness; the menstrual secretion was also defective. By a continuance of the same remedies during two months every vestige of the complaint was permanently removed.

$\mathrm{C}_{\text {ASE }}$ 5.-This was an instance in which the disease was allowed to terminate in ulceration bcfore the patient presented herself to my notice.

Nov. 16th, 1846.-M. B-, aged thirty, was admitted a patient at the Western Dispensary, under my care, with a painful ulceration of the breast, adjacent to which were several small, indurated glandular tumours. The ulcer was small, but exceedingly painful and irritable; it produced a copious discharge of thick matter, and had defied all attempts to heal it many months before the patient was admitted. Among the varions remedies applied were different kinds of poultices, ointments, and lotions. Menstruation was imperfect. The pain felt by the patient was of the aching kind, which is one of the characters of the lymphatic tumour. I prescribed pills, composed of sulphate of iron and aloes, which I directed to be taken twice a day, and I ordered the continuance of an evaporating poultice, which had before been applied, and afforded relief.

On Dec. 14th, the improvement observed was astonishing; the ulcer had healed, the lymphatic glands were greatly diminished, and all pain had subsided.

1847, Jan. 7th.-The patient was discharged, every symptom of the disease having disappeared.

CASE 6.-The diagnosis in this case, as I have observed before, was rendered obscure by the natural size of the breast, which exceeded the ordinary limits.

1838, April 23rd.-A lady, aged forty-three, residing in the country, consulted me respecting a tumour in the right breast, which had been a source of pain and apprehension during twelve months. She had been under the care of a general practitioner and an eminent provincial surgeon, without obtaining any relief or accurate information respecting the nature of the tumour, which she was led to believe was of a malignant character. The swelling was as large as a walnut, irregular on its surface, exceedingly tender and painful on pressure, and seated in the upper part of the breast, in the conrse of the absorbents. Every three or four months the uterus discharged a kind of false membrane, instead of the proper menstrual secretion. The bowels were habitually relaxed; the axilla was free from disease; the pain in the tumour was described as an aching, and sometimes as a stinging sensation. On a careful examination, which was much impeded by the mass of cellular and adipose membrane beneath the integuments, $I$ discovered in the irregular surface of the tumour the enlargement and inclination of the lymphatic vessels, which are characteristic of this disease, and I therefore gave my patient a favourable opinion and prognosis, notwithstanding that some retraction of the nipple, quite unusual in connexion with this kind of tumour, had taken place. I prescribed two grains of sulphate of iron twice a day, in conjunction with tincture of colchicum, which I was induced to combine with the steel, on account of a manifest gouty diathesis in the constitution.

May 5 th.-The tumour much smaller and more free from pain; the catamenia has appeared naturally, nipple less 
retracted, and the lvmphatic character of the tumour more evident.

12th.-All pain removed, and the swelling in the breast rapidly diminishing.

August 18th.-No return of pain, except on the approach of menstruation. No remains of the tumour left. The medicines, which had been taken regularly up to this time, were now discontinued.

1839, July 6th.-I was again consulted, on account of an attack of rheumatism in the muscles of the chest and shoulder, which was soon removed by appropriate treatment. This attack was, however, followed by a slight return of the lymphatic tumour, observable in the breast on the 12 th. A repetition of the medicines prescribed when I was first consulted, permanently removed the tumour, and the patient has continued free from the disease to the present time.

Chester-square, London, June, 1847.

\section{ZRebiems.}

Lectures on Diseases of the Eye. By JoHn Morgan, F.L.S., late Surgeon of Guy's Hospital, \&c. Second edition, care fully revised and enlarged, with notes, by JoHN F. France, Surgeon of the Eye Infirmary, and Lecturer on Ophthalmic Surgery at Guy's Hospital. London : Highley. 1848. 8vo, pp. 222 .

WE have always regarded ophthalmic surgery as one of the most interesting and difficult subjects in the range of studies the medical practitioner has to follow; and although we think that in this department much less can be learned from books and written descriptions than in most other walks of professional study, and that in order to gain a correct knowledge of diseases of the eye, the student must trust especially to the book of Nature, yet he may be much facilitated in his pursuit by making himself carefully acquainted with the recorded experience of those who have been conspicuous in this department of medical science. We therefore hail with pleasure the work of the late Mr. Mrorgan,consisting of a series of lectures delivered by him at Guy's Hospital, carefully revised by Mr. France, who has added notes of his own on most of the subjects, and has prefaced the work by a memoir of the deceased anthor.

A great portion of the book is taken up by the consideration of diseases of the conjunctivæ, and the symptoms and treatment of their various forms are clearly and pretty fully dwelt upon. In the chapter on aphthous inflammation of the conjunctivæ, so very common amongst children in large towns, the author dwells upon a point which is not unfrequently overlooked by practitioners-namely, the ill effects which result from neglected treatment.

"If the disease be neglected, particularly when occurring in a strumous or cachectic subject, ulcerative infiammation is almost invariably set up, and extends to the subjacent cornea or anterior part of the sclerotic. Thus, from neglected aphthous ophthalmia, it is by no means uncommon to lave a spreading ulcer of the cornea produced."-p. 30 .

Great caution must be employed by the reader in adopting the views of the author with respect to the treatment of purulent ophthalmia of adults, as he lays down rules which, if always acted upon, must prove injurious. It is true that the purulent ophthalmia of adults, produced by the gonorthcal poison, is one of the most rapidly destructive diseases to the organ of vision, and therefore demands being combated by powerful remedies; but it must be evident to every one who has any considerable experience in diseases of the eye, that the large depletions recommended cannot, in a great many cases, be safely carried out. We are glad to see that the editor, in a lengthy note, particularly enjoins caution in this respect, and refers to the improvement which the late Mr. Tyrrell introduced in the treatment of this disease, and to the opinion formed by that gentleman as to the cause of the destruction of the cornea-viz., its not being from intense inflammation, but from the pressure of the chymosed conunctiva upon the cornea, impeding its nutrition.
The symptoms of strumous ophthalmia are well and clearly depicted. The author considers that strumous ophthalmia begins in irritability of the retina, and that subsequently the ther parts of the eye are involved in the inflammation, and he assumes that the retina is the seat of the great intolerance of light, so remarkable in this disease. The editor, as we think, more correctly ascribes it to the conjunctiva itself, and not to the retina.

We are of opinion that the author speaks too disparagingly of blisters in the following passage:-

"Be, careful, above all, not to apply blisters or counterirritants, if you can possibly avoid it, for though the application of a blister is sometimes serviceable, yet the highly irritable state of the absorbent system in strumous children frequently gives rise to inflammation and suppuration of the cervical glands in consequence."-p. 84.

In treating affections of the cornea, the author says that opacities of that membrane, if not of long standing, may be got rid of by proper treatment. From an idea that chronic opacity of the cornea may not be dissipated, practitioners are in the habit of doing nothing, and thus vision becomes much impaired. Much may certainly be done by judicious treatment, and a valuable guide for prognostic and treatment will be found in the existence or non-existence of vessels leading to the opacity: if the former obtain, we may with reason hope that benefit will be derived from the use of mercury and local stimulants; but if there be no apparent vascular supply to the disease, we must not look for improvement under any form of treatment.

The author speaks as highly of mercury in the treatment of syphilitic iritis as it deserves, but we think that there is not sufficient caution expressed with respect to the administra. tion of this powerful remedy. There are many cases of the disease occurring in persons whose system has been broken down by various causes, and where it is necessary to be circumspect in the use of mercury. In such it may be com. bined with quinine; and by this means the system can be well affected without detriment to the patient. The editor very properly puts the reader on his guard against adopting the instructions of the author with respect to indiscriminate bleeding in this disease.

"He feels called upon, however, to give his opinion, the result of an extensive experience, that, in the treatment of this disease, general bloodletting is very rarely demanded, or even admissible; and that the withdrawal of from six to twelve ounces of blood from the temple by the cupping-glass, (the application of which may, if necessary, be renewed) will almost invariably suffice. Hale, robust stibjects of this dis. temper in ay be benefited by venesection, but such cases are quite exceptional."-p. 139.

Mr. Morgan's experience has led him to the conviction that the removal of a melanotic eye, when the disease has become fully developed, and the constitution has suffered, is totally useless. Our own experience on this point entirely agrees with this. We have thays had the misfortune to see the disease return after operation, or the patient die with the morbid degeneration developed in various parts of the body.

That portion of the work is meagre which treats of cataract and the operations for its relief. Mnch that is highly practical and absolutely necessary for the ophthalmic student is not even hinted at. We are astonished, too, that those general conditions contra-indicating the operations for cataract are not given. The diagnosis of the most common forms of this disease would certainly have been useful; indeed, we may say they are imperatively required in such a book. It is " essentially necessary" to prepare a patient for the operation, and the only direction given is that of "reducing the vascular action below the standard of health." This is uncertain advice, and would lead us to infer that a patient cannot be lowered too much for the due success of an operation. It is now a wellestablished fact, that many patients require to be "prepared" for the operation by bringing up their strength, and not pulling it down. Non-adhesion of the section of the cornea is 